\title{
Predictive Significance of IL-17A Serum Levels during Chemotherapy in Senegalese Women with Cervical Cancer
}

\author{
Folly Mawulolo Gaba1', Babacar Mbengue ${ }^{1 *}$, Maimouna Diop¹, Doudou Diouf ${ }^{2}$, Sidy Ka ${ }^{2}$, \\ Doudou Georges Massar Niang1, Ramatoulaye Ndiaye', Ibrahima Mounkeila Seydou², \\ Jean Pascal Demba Diop ${ }^{3}$, Moustapha Mbow ${ }^{1}$, Rokhaya Ndiaye Diallo3, \\ Maguette Sylla Niang1, Mbacké Sembene ${ }^{4}$, Ahmadou Dem², Alioune Dieye ${ }^{1}$
}

\author{
${ }^{1}$ Service d'Immunologie, Faculté de Médecine, de Pharmacie et d'Odontologie, Université Cheikh Anta Diop (UCAD) de Dakar, \\ Dakar, Sénégal \\ ${ }^{2}$ Service de Cancérologie, Institut Juliot Curie, Hôpital Aristide Le Dantec, Dakar, Sénégal \\ ${ }^{3}$ Service de Génétique Humaine, Faculté de Médecine, de Pharmacie et d’Odontologie, UCAD, Dakar, Sénégal \\ ${ }^{4}$ Département de Biologie Animale, Faculté des Sciences et Techniques, UCAD, Dakar, Sénégal \\ Email: *babacar.mbengue@ucad.edu.sn
}

How to cite this paper: Gaba, F.M., Mbengue, B., Diop, M., Diouf, D., Ka, S., Niang, D.G.M., Ndiaye, R., Seydou, I.M., Diop, J.P.D., Mbow, M., Diallo, R.N., Niang, M.S., Sembene, M., Dem, A. and Dieye, A. (2019) Predictive Significance of IL-17A Serum Levels during Chemotherapy in Senegalese Women with Cervical Cancer. Open Journal of Immunology, 9, 37-47. https://doi.org/10.4236/oji.2019.94004

Received: December 17, 2019

Accepted: December 27, 2019

Published: December 30, 2019

Copyright ( 2019 by author(s) and Scientific Research Publishing Inc. This work is licensed under the Creative Commons Attribution International License (CC BY 4.0).

http://creativecommons.org/licenses/by/4.0/

\section{(c) (i) Open Access}

\begin{abstract}
Background and Objectives: Cervical cancer is a leading cause of cancer death in female populations. It is a virally induced carcinoma resulting from sexually transmitted high risk Human Papillomavirus infections (e.g. HPV-16, HPV-18). Previous studies have shown associations between IL-17A levels in cancer micro-environments and metastasis of tumor cells. In Africa, chemotherapy (CT) is the standard first-line treatment for cervical cancer and the prognosis remains poor for metastatic and recurrent cases. The impact of CT as a treatment option is still unclear. We investigated the prognostic relevance of IL-17A profiles in Cervical cancer patients (CP) patients treated with cisplatin in combination with 5 -fluouracil $(5 \mathrm{FU})$ for three cycles. Methods: The study included $57 \mathrm{CP}$ and 59 women with no history of malignancy as healthy controls (HC). IL-17A plasma levels were evaluated by ELISA. For each CP, three blood samples were collected at three-week intervals before initiation of the chemotherapy protocol. Results: Before chemotherapy CP showed higher serum levels of IL-17A compared to HCs $(p=$ 0.035). No relation was detected between age and IL-17A levels. We observed a significant increase in serum IL-17A during treatment of the CP group $(p<$ 0.05). Depending on chemotherapy's efficacy, $\mathrm{CP}$ were divided into 1) non responders, 2) partial responders and 3) good responders. Non-responders patients showed significantly higher serum levels of IL-17A during the follow-up compared to partial and good responder groups both following the
\end{abstract}


first CT cycle $(p<0.05)$ and the second CT cycle $(p<0.03)$. Conclusion: Our results suggest that high serum levels of IL-17A are associated with poor responses to classical chemotherapy. However, considering these results to design CC biomarkers, we need further investigations particularly about the relevant prognostic indicator following chemotherapy.

\section{Keywords}

Cervical Cancer, Chemotherapy, Interleukin 17A

\section{Introduction}

Cervical cancer is a leading cause of cancer death in female populations especially in developing countries [1]. The link between cervical cancer and oncogenic human papillomavirus (HPV) infection has been clearly established but some additional factors must contribute to pathogenesis leading to persistence of lesions and progression to malignancy [2]. Despite advances in screening and HPV vaccination, a significant number of women develop advanced disease particularly in low incoming countries. In Africa, patients are often very young presenting highly aggressive forms of cervical cancer including multiple nodules and metastatic stages associated with rapid fatal evolution [3]. Contributing immunological factors are poorly understood in cervical cancer. Previous studies indicate that $\mathrm{T}$ lymphocytes release soluble factors such as cytokines that may be implicated in cancer development [4] [5] [6] and some T-cell sub-groups appear to play a crucial role in tumor progression [7] [8], including Th17 cells characterized by IL-17 release. IL-17 is a pro-inflammatory cytokine with six previously described homologues (IL-17 A - F $_{\text {) }}$ of which IL-17A is the prototype with the highest degree of homology to other members [9]. Pro or anti-tumor effects of IL-17A have been attributed to inflammation in tumor micro-environments [10] and several studies have implicated its involvement in cancer growth [11]. IL-17A release induces mediators that promote neutrophils recruitment and proliferation [12] [13].

Classical options for cervical cancer management include surgery, radiation therapy, chemotherapy (CT) or combinations of these interventions [14]. In Africa, CT is the standard first-line treatment for cervical cancer although the prognosis is poor for metastatic and recurrent forms [15]. The impact of CT on the immune response to tumor cells is still unclear although some previous studies have reported a hyper-stimulation of anti-tumoral activity during CT. These effects seem to depend on certain cytokines which have been widely investigated in new immunotherapeutic approaches to cancer management [15]. The development of new strategies to combat cervical cancer which combine immunotherapy and CT, and are adapted to clinical phenotypes encountered in African women, is urgently needed. However, these therapeutic advances should be based both on a better knowledge of factors inducing cancer growth and metas- 
tasis, and on comprehensive patient follow-up involving clinical and biological parameters that have prognostic relevance to treatment response.

In this study, we quantified IL-17A levels in the sera of Senegalese cervical cancer patients and healthy controls and evaluated the relevance of this biomarker as a prognostic indicator of patient response to classical CT.

\section{Materials and Methods}

\subsection{Patients, Samples and Recruitment}

This study included 57 Senegalese cervical cancer patients (CP) and 59 healthy controls (HC) with no history of malignancy. The study was performed at the Cancer Department of the Juliot Curie Institute (hospital Aristide Le Dantec, Dakar, Senegal) from July to October in two successive years 2017 and 2018.

Recruited CP were recently diagnosed for cervical cancer and had not undertaken any CT or radiotherapy. The major exclusion criteria included prior treatment with traditional plant-based remedies and bacterial or viral infections such as tuberculosis, HIV and hepatitis B. These infectious diseases were systematically investigated by the medical team responsible for the monitoring. All selected patients were managed by the same medical staff and the CT protocol was in accordance with Senegalese national recommendations specifying one cycle every 21 days. Usually, three cycles are recommended before undertaking a thorough clinical examination to evaluate therapy response. Each patient followed a treatment protocol depending on individual clinical disease characteristics and general health status. Overall for selected patients, cisplatin was used in combination with 5-fluouracil (5-FU). In terms of doses, cisplastin was administrated $50-75 \mathrm{mg} / \mathrm{m}^{2}$ of body surface every 3 weeks and 5-FU was used with to $1000 \mathrm{mg} / \mathrm{m}^{2}$ every 3 weeks for 3 cycles.

Selected HC are women free of known tumors or chronic inflammatory diseases. They live in Dakar and were followed for high blood pressure in the Medical Center of the Faculty of Medicine, more than two years with biological analyses every three months.

Peripheral blood from all study participants (CP and HC) were sampled using EDTA vacutainers. For CP, sample collection was based on the treatment protocol. Three samples were collected per CP, corresponding to one before each CT cycle and are designated respectively: Sample 1 (S1), Sample 2 (S2) and Sample 3 (S3). However, only one sample was obtained per HC. All samples were immediately centrifuged and sera supernates were stored at $-80^{\circ} \mathrm{C}$.

\subsection{Ethics Statement and Procedure}

This study was performed at Hospital Aristide Le Dantec in Dakar (Senegal) in the internal institute dedicated to cancer. Immunological analyses were performed in the Laboratory of Immunology in the Faculty of Medicine of Cheikh Anta Diop University in Dakar. Informed consent was obtained from each par- 
ticipant and/or relatives prior to inclusion, after providing written or verbal information in their native language. The protocol 0297/2018/CER/UCAD was approved by Institutional Ethics Committee of Cheikh Anta Diop University (Dakar, Senegal).

\subsection{Determination of Serum IL-1A Levels}

IL-17A levels were measured by ELISA kits ready SET-GO ${ }^{\circledR}$ (Affymetrix eBioscience Inc., San Diego, CA, USA) according to the manufacturer's instructions. Briefly, the lyophilized standard was reconstituted to obtain a stock at $500 \mathrm{pg} / \mathrm{ml}$. and serially diluted to generate a standard curve. $100 \mu \mathrm{l}$ of diluted capture antibody was added to each well of the microtiter plate. Plates were incubated overnight at $4^{\circ} \mathrm{C}$. After washing, diluted standards or samples $(100 \mu \mathrm{l})$ were added and plates were incubated $2 \mathrm{hr}$ at room temperature. After washing, $100 \mu \mathrm{l}$ of the detection antibody was added to each well and incubated $1 \mathrm{hr}$ at room temperature. Avidin-HRP $(100 \mu \mathrm{l})$ was added to each well and incubated $30 \mathrm{~min}$ at room temperature, followed by the addition of $100 \mu \mathrm{l}$ of substrate for $15 \mathrm{~min}$ and $50 \mu \mathrm{l}$ of stop solution. Optical densities were immediately determined using a microplate reader set to $450 \mathrm{~nm}$. The results are expressed in $\mathrm{pg} / \mathrm{ml}$.

\subsection{Statistical Analysis}

The data were analyzed with Statview ${ }^{\circledR}$ 5.1. Software. To compare IL-17A levels between groups, the non-parametric Mann Whitney and Kruskal-Wallis tests were used. Correlations were evaluated by using Spearman rank test and $p<0.05$ values were considered to be significant.

\section{Results}

\section{Characteristics of the study population}

This study included 57 Senegalese patients (CP) and 59 healthy women without a history of malignancy as controls ( $\mathrm{HC}$ ). Although the mean age of CPs differed significantly from $\mathrm{HCs}$, there was no relationship between age and IL-17A levels. CP and HC data are summarized in Table 1.

Clinically, $91.2 \%$ of the tumors were squamous cell carcinoma and $8.8 \%$ were adeno-carcinoma type. According to the FIGO classification, stages I and II were found in $38.6 \%$ of patients and stages III-IV were encountered in $43.9 \%$ of patients (Table 2).

Comparison of IL-17A levels between HC and CP before chemotherapy and in $\mathrm{CP}$ during treatment

Prior to chemotherapy, the highest levels of IL-17A were observed in CP compared to HC (median $2.62 \mathrm{pg} / \mathrm{ml}$ versus $5.51 \mathrm{pg} / \mathrm{ml} ; p=0.035$ ) (Figure 1 (a)). Figure 1(b) shows the comparison of IL-17A levels before each of the three CT cycles (S1, S2, and S3). The results show a significant increase in IL-17A levels at S3 (median $9.71 \mathrm{pg} / \mathrm{ml})(p<0.05)$ compared to both S1 (median $5.51 \mathrm{pg} / \mathrm{ml})$ and $\mathrm{S} 2$ (median $5.80 \mathrm{pg} / \mathrm{ml}$ ). 


\section{Profiles of IL-17A levels during CT treatment in patients}

We were also interested in investigating possible relations between IL-17A levels during chemotherapy treatment. A positive correlation was found between the IL-17A levels for S1 and S2 $(p<0.001$, rho $=0.81$, Spearman rang test $)$ (Figure 2(a)) and between S2 and S3 ( $p=0.011$, rho $=0.80$, Spearman rang test) (Figure 2(b)).

Depending on CT efficacy, patients were divided into three groups: 1) CP Table 1. Biological characteristics of the study population.

\begin{tabular}{cccc}
\hline Characteristics & CC Patients $(\mathrm{N}=57)$ & Healthy Controls $(\mathrm{N}=59)$ & $p$ \\
\hline Age (min-max) years & $43.8(35-69)$ & $37.4(20-60)$ & $<0.01$ \\
Lymphocytes $\left(10^{3} / \mu \mathrm{l}\right)$ & $2.2(0.2-8.6)$ & $2.2(1.8-3.1)$ & 0.37 \\
Polynuclear cells $\left(10^{3} / \mu \mathrm{l}\right)$ & $10.3(1.8-80.6)$ & $1.9(1.2-2.7)$ & $<0.001$ \\
Monocytes $\left(10^{3} / \mu \mathrm{l}\right)$ & $0.9(0.1-8)$ & $0.5(0.3-1.4)$ & 0.11 \\
Red Blood Cells $\left(10^{6} / \mu \mathrm{l}\right)$ & $4.9(2.1-14.5)$ & $4.6(4.1-5.3)$ & 0.59 \\
Hemoglobin $(\mathrm{g} / \mathrm{l})$ & $11.2(6.8-13.9)$ & $12.7(11.8-15.0)$ & 0.07 \\
Hematocrit $(\%)$ & $37.3(23.7-68)$ & $37.4(36.4-39.7)$ & 0.68 \\
\hline
\end{tabular}

Legends: $\min =$ minimum, $\max =$ maximum, $\mathrm{N}=$ number of patients. Data were determined by the hospital's medical laboratory. $p=p$-value of comparison between $\mathrm{CP}$ and HC groups with Mann Whitney rank test.

Table 2. Histological, gynecological and therapeutic distribution of cervical cancer patients.

\begin{tabular}{|c|c|}
\hline Characteristics & $\mathrm{N}(\%)$ \\
\hline \multicolumn{2}{|l|}{ Histological data } \\
\hline Squamous cell carcinoma & $52(91.2)$ \\
\hline Adenocarcinoma & $5(8.8)$ \\
\hline \multicolumn{2}{|l|}{ FIGO Stage } \\
\hline I - II & $22(38.6)$ \\
\hline II - IV & $25(43.9)$ \\
\hline $\mathrm{nd}^{*}$ & $10(17.5)$ \\
\hline \multicolumn{2}{|l|}{ Parity } \\
\hline 0 & $5(8.8)$ \\
\hline $1-5$ & $26(45.6)$ \\
\hline $5-10$ & $22(38.6)$ \\
\hline$>10$ & $4(7.0)$ \\
\hline \multicolumn{2}{|l|}{ Gestity } \\
\hline 0 & $6(10.5)$ \\
\hline $1-5$ & $23(40.4)$ \\
\hline $5-10$ & $24(42.1)$ \\
\hline$>10$ & $4(7.0)$ \\
\hline \multicolumn{2}{|l|}{ Responses to chemotherapy } \\
\hline Good responders & $13(22.8)$ \\
\hline Partial responders & $19(33.3)$ \\
\hline No responders & $17(29.8)$ \\
\hline$n d^{*}$ & $8(14.1)$ \\
\hline
\end{tabular}

*data not available. 

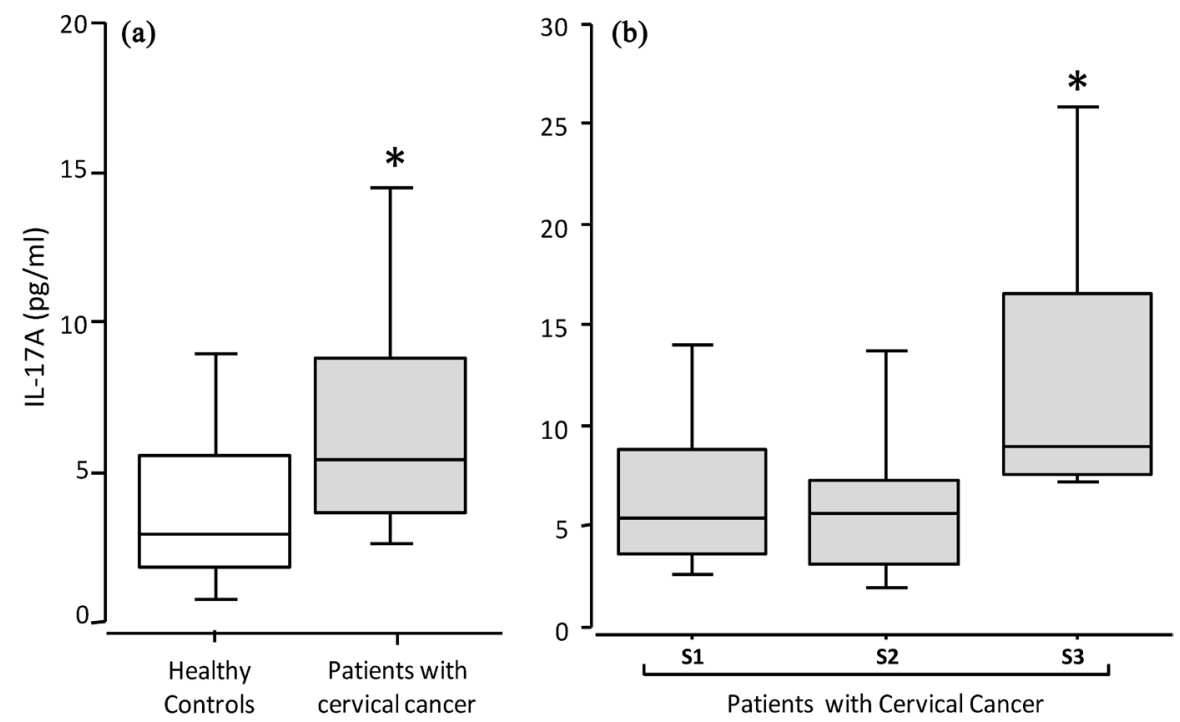

Legends: The evaluation of IL-17A levels during the different chemotherapy courses showed significantly higher IL-17A levels after the second course of chemotherapy compared to pre-treatment and post-first course levels. ${ }^{\star} p<0.05 \mathrm{~S} 1=$ Sample 1 (collected at day 0 ), $\mathrm{S} 2=$ Sample 2 (collected at day 21) and S3 = Sample 3 (collected at day 42) Comparison of IL-17A levels between controls and patients shows a statistically significant difference with higher IL-17A levels in patients.

Figure 1. Comparison of IL-17A levels between patients and healthy controls (a) and in the group of patients according to the day of sampling (b).
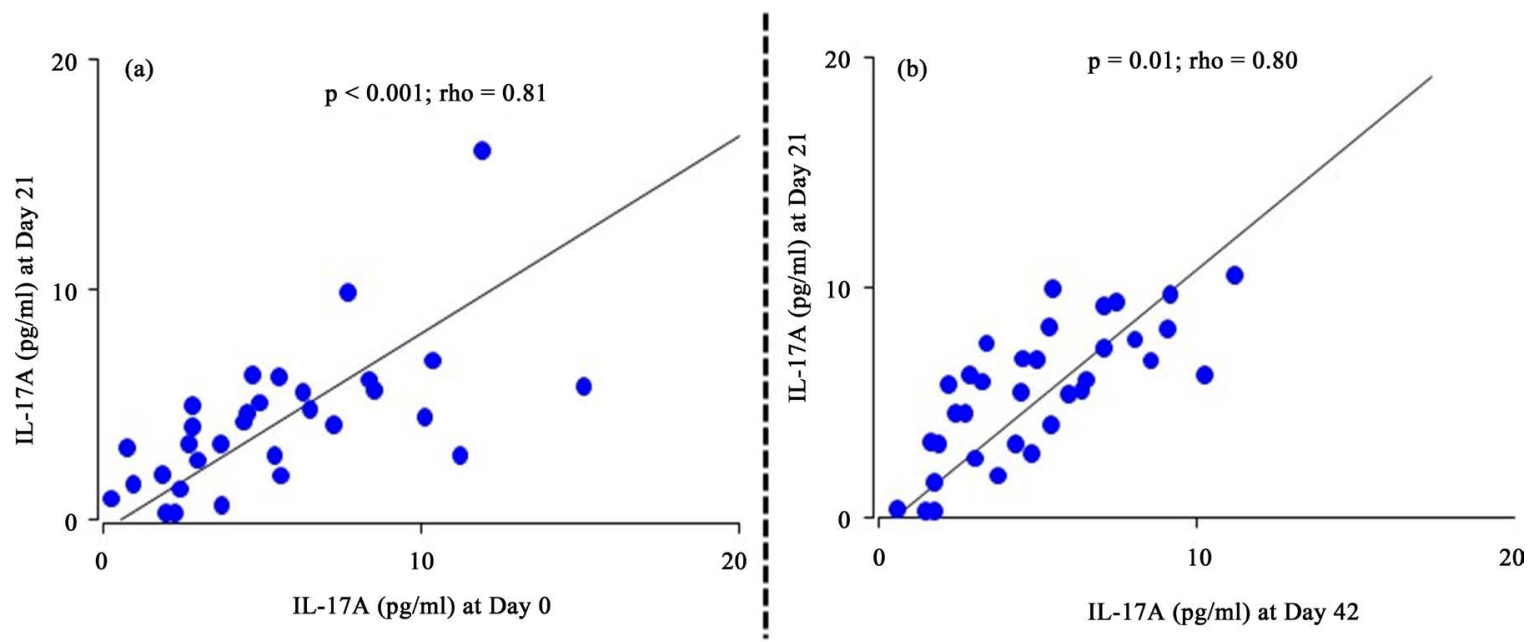

Legends: Day 0 is the day before any chemotherapy. The first course of chemotherapy was administrated at day 0 and Sample 1 (S1) was taken before this treatment. At 21 days, blood sample 2 (S2) was collected before the second cycle of chemotherapy. Sample 3 or S3 was collected at day 42 days before the third cycle. IL-17A levels are positively correlated during treatment $(p<0.05$, rho $\geq 0.80)$. Results were analyzed using the Spearman rank test.

Figure 2. Relationship between IL-17A levels during the three cycles of chemotherapy (a) between day 0 (S1) and day 21 (S2); (b) between day 21 (S1) and day 42 (S3).

non-responders showing an increase of the tumor size or absence of variation associated to metastasis $(\mathrm{N}=17)$ 2) $\mathrm{CP}$ partial responders considered as patients with a $50 \%-70 \%$ of decrease in baseline tumor size $(\mathrm{N}=19)$ and 3) $\mathrm{CP}$ good responders that are women with a decrease more than $70 \%(\mathrm{~N}=13)$. Between these three groups; IL-17A levels were compared. Figure 3 shows that CT 


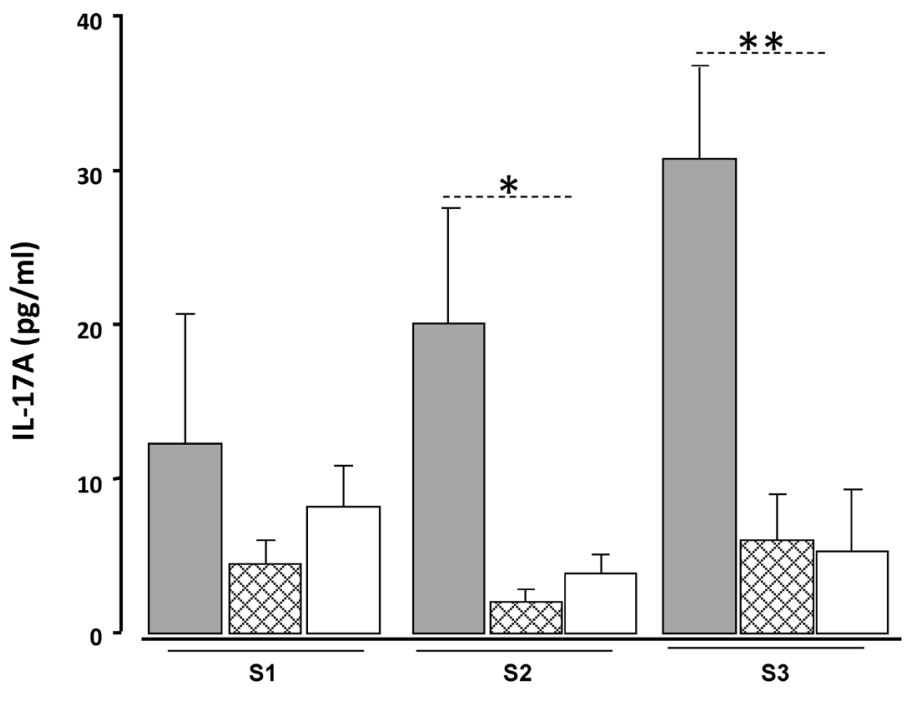

No responders (17) $\bigotimes$ Partial responders (19) $\square$ Good responders (13)

Legends: ${ }^{\star} 0.01<p<0.05 ;{ }^{*} 0.01 \leq p, \mathrm{~S} 1=$ Sample 1 (collected at day 0 ), $\mathrm{S} 2=$ Sample 2 (collected at day 21 ) and S3 = Sample 3 (collected at day 42 ).

Figure 3. Evolution of IL-17A levels depending on response to treatment in CC patients.

non-responders had significantly higher levels of IL-17A compared to partial and good responders both after the first CT cycle (S2, $p=0.032)$ and the second $\mathrm{CT}$ cycle $(\mathrm{S} 3, p=0.027)$. Although no similar significant differences were observed before initiation of CT (S1), there does seem to be a trend towards higher IL-17A levels in cervical cancer patients who were subsequently determined to be non-responders.

\section{Discussion}

In West Africa, the early onset of cervical cancer and difficulties with cancer management are aggravated by a lack of diagnostic and prognostic biomarkers. This study attempts to address this problem by identifying relevant immunological biomarkers. Our main objective was to evaluate the impact of CT on IL-17A levels in the peripheral blood of Senegalese women with cervical cancer. There was close collaboration between clinicians and researchers during the recruitment of patients and controls: (i) patients were women recently diagnosed with cervical cancer and subsequently treated by CT and (ii) controls were women with no history of malignancy of any type.

In this study, we observe that the majority of patients were diagnosed during the late stage disease (III-IV). It is a common observation in developing countries [16]. In these areas, patients often consult relatively late, when the cancer is already at a very advanced stage. We also found that the majority of patients had squamous cell carcinoma type cancer (91.2\%); these results are in line with those found in several studies conducted among the Senegalese population [17] [18].

IL-17A is an important contributing cytokine in the development of many inflammatory diseases and it is also frequently detected in tumor microenviron- 
ments [19] [20] [21]. However, little is known about possible effects of IL-17A on cervical cancer progression. Souza et al. have documented correlations of IL-17 concentration in serum from patients with different grades of squamous intraepithelial lesions and invasive cervical carcinoma [11], but did not evoke a role for IL-17A in the prognosis of cervical cancer. Here, we document the presence of higher concentrations of IL-17A in the sera of untreated cervical cancer patients compared to controls. Similarly, Zhang et al. have shown that IL-17 was highly expressed in patients with gastric cancer [22] and a significant role for IL-17 in ovarian tumor growth and angiogenesis has been reported [23]. Our results are consistent with these previous studies and together they strongly implicate IL-17 in the development of malignant tumors.

Lyon et al. showed a significant increase of IL-17A levels in the sera of patients newly diagnosed with breast cancer compared to healthy women [24]. This increase could also be explained by the existence of local inflammation at the tumor site, resulting in the secretion not only of IL-17, but also of other cytokines such as IL-6, also considered to play a role in tumor growth [12]. IL-17A production by Th17 cells appears to be dependent on IL- 6 released by CPA [25], and IL-17A inducing inflammation has been shown to be promoted by high levels of IL- 6 and low TGF- $\beta$ release, both of which are pro-tumoral cytokines with directs actions [26].

Our results showed a slight increase of IL-17A levels after the CT cycles with significant variation between CPs and HCs. This result supports the notion of IL-17A production by Th17 cells (TIL) indirectly influenced by anticancer drugs [27]. This release is only weakly reflected at the serum level, hence the importance of characterizing intra-tumor Th17 lymphocytes producing this cytokine. The concept of immuno-contexture recently developed in the evaluation of the prognostic relevance of intra-tumor immune cells (Th1 and $\mathrm{TCD} 8^{+}$), supports this interpretation and the interest of monitoring Th17 cells present in tumor tissue [28]. Our data showing significantly higher IL-17A levels in Non-responder patients compared to partial and good responder patients following the first and second CT cycles is also consistent with this perspective and further supports the pro-tumor role of IL-17A [29]. This finding suggests that a possible reorientation of the immune response in the tumor microenvironment away from Th17 and/or Th2 in favor of Th1 and/or TCD8 responses might be protective.

\section{Conclusion}

Based on our work, a majority of histological types of cervical tumors in Senegalese patients were squamous carcinoma. Moreover, we did not found a difference in cytokines levels according to the clinical or histological data. However, compared to healthy controls we highlighted a significant increase of IL-17A levels in cervical cancer patients particularly in Non-responders individuals. Consequently, our findings suggest that treatment failure during chemotherapy would be characterized by an increase of plasmatic IL-17A levels, but the use of 
IL-17A as a prognostic biomarker during the treatment requires further investigations, such as the evaluation of cytokines balance with PBMC Intracellular Staining and some genetic explorations.

\section{Acknowlegements}

The authors are grateful to the patients and their relatives for the active participation and continued collaboration in this work. The authors acknowledge Dr. Shirley Longacre (Vaximax, Paris), G. Diop (UCAD, Dakar), F. Thiam (ESP, Dakar) for constant and helpful support and A. Soumah Sall, A. Thiam and M. Faye for expert technical assistance.

\section{Conflicts of Interest}

The authors declare no conflicts of interest regarding the publication of this paper.

\section{References}

[1] Siegel, R.L., Miller, K.D. and Jemal, A. (2017) Cancer Statistics, 2017. CA: A Cancer Journal for Clinicians, 67, 7-30. https://doi.org/10.3322/caac.21387

[2] Schiffman, M.H. and Brinton, L.A. (1995) The Epidemiology of Cervical Carcinogenesis. Cancer, 76, 1888-1901. https://doi.org/10.1002/1097-0142(19951115)76:10+<1888::AID-CNCR2820761305 >3.0. $\mathrm{CO} ; 2-\mathrm{H}$

[3] Ferlay, J., Soerjomataram, I., Ervik, M., Dikshit, R., Eser, S., Mathers, C., Rebelo, M., Parkin, D., Forman, D. and Bray, F. (2013) Cancer Incidence and Mortality Worldwide: IARC Cancer Base No. 11.

[4] Shankaran, V., Ikeda, H., Bruce, A.T., White, J.M., Swanson, P.E., Old, L.J. and Schreiber, R.D. (2001) IFNgamma and Lymphocytes Prevent Primary Tumour Development and Shape Tumour Immunogenicity. Nature, 410, 1107-1111. https://doi.org/10.1038/35074122

[5] Janssen, E.M., Droin, N.M., Lemmens, E.E., Pinkoski, M.J., Bensinger, S.J., Ehst, B.D., Griffith, T.S., Green, D.R. and Schoenberger, S.P. (2005) CD4 ${ }^{+}$T-Cell Help Controls $\mathrm{CD}^{+}$T-Cell Memory via TRAIL-Mediated Activation-Induced Cell Death. Nature, 434, 88-93. https://doi.org/10.1038/nature03337

[6] Marzo, A.L., Kinnear, B.F., Lake, R.A., Frelinger, J.J., Collins, E.J., Robinson, B.W.S. and Scott, B. (2000) Tumor-Specific CD4 ${ }^{+}$T Cells Have a Major "Post-Licensing" Role in CTL Mediated Anti-Tumor Immunity. The Journal of Immunology, 165, 6047-6055. https://doi.org/10.4049/jimmunol.165.11.6047

[7] Street, S.E., Cretney, E. and Smyth, M.J. (2001) Perforin and Interferon-Gamma Activities Independently Control Tumor Initiation, Growth, and Metastasis. Blood, 97, 192-197. https://doi.org/10.1182/blood.V97.1.192

[8] Street, S.E., Trapani, J.A., MacGregor, D. and Smyth, M.J. (2002) Suppression of Lymphoma and Epithelial Malignancies Effected by IFN Gamma. Journal of Experimental Medicine, 196, 129-134. https://doi.org/10.1084/jem.20020063

[9] Kolls, J.K. and Linden, A. (2004) Interleukin-17 Family Members and Inflammation. Immunity, 21, 467-476. https://doi.org/10.1016/j.immuni.2004.08.018

[10] Zou, W. and Restifo, N.P. (2010) $\mathrm{T}_{\mathrm{H}} 17$ Cells in Tumour Immunity and Immuno- 
therapy. Nature Reviews Immunology, 10, 248-256.

https://doi.org/10.1038/nri2742

[11] Souza, J.M., Matias, B.F., Rodrigues, C.M., Murta, E.F. and Michelin, M.A. (2013) IL-17 and IL-22 Serum Cytokine Levels in Patients with Squamous Intraepithelial Lesion and Invasive Cervical Carcinoma. European Journal of Gynaecological Oncology, 34, 466-468.

[12] Cua, D.J. and Tato, C.M. (2010) Innate IL-17-Producing Cells: The Sentinels of the Immune System. Nature Reviews Immunology, 10, 479-489. https://doi.org/10.1038/nri2800

[13] Pelletier, M., Maggi, L., Micheletti, A., Lazzeri, E., Tamassia, N., Costantini, C., Cosmi, L., Lunardi, C., Annunziato, F., Romagnani, S. and Cassatella, M.A. (2010) Evidence for a Cross-Talk between Human Neutrophils and Th17 Cells. Blood, 115, 335-343. https://doi.org/10.1182/blood-2009-04-216085

[14] Marchetti, C., De Felice, F., Di Pinto, A., Romito, A., Musella, A., Palaia, I., Monti, M., Tombolin, V., Muzii, L. and Benedetti Panici, P. (2018) Survival Nomograms after Curative Neoadjuvant Chemotherapy and Radical Surgery for Stage IB2-IIIB Cervical Cancer. Cancer Research and Treatment, 50, 768-776.

https://doi.org/10.4143/crt.2017.141

[15] Mallmann, P. (2016) A Change of Paradigm in the Treatment of Cervical Cancer. Oncology Research and Treatment, 39, 500. https://doi.org/10.1159/000448967

[16] Waggoner, S.E. (2003) Cervical Cancer. The Lancet, 361, 2217-2225. https://doi.org/10.1016/S0140-6736(03)13778-6

[17] Ndiaye, R., Dem, A., Mbaye, P.M., Guèye, P.M., Diop, G., Diop, P.A. and Faye, O. (2014) Étude du codon 72 du gène p53 dans la prédisposition au cancer du col de l'utérus au Sénégal. Bulletin du Cancer, 101, 789-794.

[18] Dem, A., Dieng, M.M., Traore, B., Gaye, M., Diop, M. and Touré, P. (2008) Les carcinomes épidermoïdes du col utérin à l'Institut du cancer de Dakar. Cahiers d'études et de recherches francophones/Santé, 18, 31-33. https://doi.org/10.1684/san.2008.0094

[19] Zhu, X., Mulcahy, L.A., Mohammed, R.A., Lee, A.H., Franks, H.A., Kilpatrick, L., Yilmazer, A., Paish, E.C., Ellis, I.O., Patel, P.M. and Jackson, A.M. (2008) IL-17 Expression by Breast-Cancer-Associated Macrophages: IL-17 Promotes Invasiveness of Breast Cancer Cell Lines. Breast Cancer Research, 10, R95. https://doi.org/10.1186/bcr2195

[20] Fujino, S., Andoh, A., Bamba, S., Ogawa, A., Hata, K., Araki, Y., Bamba, T. and Fujiyama, Y. (2003) Increased Expression of Interleukin 17 in Inflammatory Bowel Disease. Gut, 52, 65-70. https://doi.org/10.1136/gut.52.1.65

[21] Li, Q., Chen, J., Liu, Y., Zhao, X., Tan, B., Ai, J., Zhang, Z., Song, J. and Shan, B. (2013) Prevalence of Th17 and Treg Cells in Gastric Cancer Patients and Its Correlation with Clinical Parameters. Oncology Reports, 30, 1215-1222. https://doi.org/10.3892/or.2013.2570

[22] Zhang, J.P., Yan, J., Xu, J., Pang, X.H., Chen, M.S., Li, L., Wu, C., Li, S.P. and Zheng, L. (2009) Increased Intratumoral IL-17-Producing Cells Correlate with Poor Survival in Hepatocellular Carcinoma Patients. Journal of Hepatology, 50, 980-989. https://doi.org/10.1016/j.jhep.2008.12.033

[23] Kato, T., Furumoto, H., Ogura, T., Onishi, Y., Irahara, M., Yamano, S., Kamada, M. and Aono, T. (2001) Expression of IL-17 mRNA in Ovarian Cancer. Biochemical and Biophysical Research Communications, 282, 735-738. https://doi.org/10.1006/bbrc.2001.4618 
[24] Lyon, D.E., McCain, N.L., Walter, J. and Schubert, C. (2008) Cytokine Comparisons between Women with Breast Cancer and Women with a Negative Breast Biopsy. Nursing Research, 57, 51-58. https://doi.org/10.1097/01.NNR.0000280655.58266.6c

[25] Bettelli, E., Korn, T., Oukka, M. and Kuchroo, V.K. (2008) Induction and Effector Functions of $\mathrm{T}_{\mathrm{H}} 17$ Cells. Nature, 453, 1051-1057.

https://doi.org/10.1038/nature07036

[26] Nam, J.S., Terabe, M., Kang, M.J., Chae, H., Voong, N., Yang, Y.A., Laurence, A., Michalowska, A., Mamura, M., Lonning, S., Berzofsky, J.A. and Wakefield, L.M. (2008) Transforming Growth Factor Beta Subverts the Immune System into Directly Promoting Tumor Growth through Interleukin-17. Cancer Research, 68, 3915-3923. https://doi.org/10.1158/0008-5472.CAN-08-0206

[27] Kryczek, I., Wei, S., Zou, L., Altuwaijri, S., Szeliga, W., Kolls, J., Chang, A. and Zou, W. (2007) Cutting Edge: Th17 and Regulatory T Cell Dynamics and the Regulation by IL-2 in the Tumor Microenvironment. The Journal of Immunology, 178, 6730-6733. https://doi.org/10.4049/jimmunol.178.11.6730

[28] Becht, E., Giraldo, N.A., Dieu-Nosjean, M.C., Sautes-Fridman, C. and Fridman, W.H. (2016) Cancer Immune Contexture and Immunotherapy. Current Opinion in Immunology, 39, 7-13. https://doi.org/10.1016/j.coi.2015.11.009

[29] Numasaki, M., Fukushi, J., Ono, M., Narula, S.K., Zavodny, P.J., Kudo, T., Robbins, P.D., Tahara, H. and Lotze, M.T. (2003) Interleukin-17 Promotes Angiogenesis and Tumor Growth. Blood, 101, 2620-2627.

https://doi.org/10.1182/blood-2002-05-1461 